\title{
Amorphous Structure and Stability of Mn Implanted GeC Ferromagnetic Semiconductor
}

\author{
C. $\operatorname{Sun}^{1}$, H. C. Floresca ${ }^{1}$, J. G. Wang ${ }^{1}$, J. Mustafa ${ }^{2}$, S. Guchhait ${ }^{2}$, D. Ferrer ${ }^{2}$, S. K. Banerjee ${ }^{2}$, G. Lian ${ }^{3}$, L. \\ Colombo $^{3}$ and M. J. Kim ${ }^{1}$ \\ ${ }^{1}$ Materials Science and Engineering, University of Texas at Dallas, Richardson, TX 75083 \\ ${ }^{2}$ Electrical and Computer Engineering, University of Texas at Austin, Austin, TX 78758 \\ ${ }^{3}$ Texas Instruments Incorporated, Dallas, TX 75243
}

Diluted magnetic semiconductors (DMS) are a family of materials where a magnetic transition element such as $\mathrm{Mn}, \mathrm{Fe}, \mathrm{Cr}$, $\mathrm{Co}$ or $\mathrm{Ni}$ is introduced into the semiconductor host to create a ferromagnetic semiconductor. Germanium, III-V compounds, silicon, and $\mathrm{ZnO}$ have attracted much attention because of the potential use as ferromagnetic semiconductors when doped with magnetic transition metals for spintronic devices [1]. The magnetic elements can be introduced during crystal growth such as in the case of molecular beam epitaxy (MBE), chemical vapor deposition (CVD) as in the case of nanowire growth or ion implantation [2-4]. Germanium manganese has also been extensively studied as in many of the DMS materials. There are mixed results and the mechanism or phase responsible for the magnetic behavior is still not clearly described, mostly because of the lack of understanding of the structure of the $\mathrm{GeMn}$ before and after annealing. When $\mathrm{Mn}$ is introduced into the host lattice of $\mathrm{Ge}$ or $\mathrm{GeC}$ at high concentrations the implantation process amorphizes the Ge and this can lead to relatively low mobility material. Our recent study shows that the magnetic properties of $\mathrm{Mn}$-implanted $\mathrm{GeC}$ were improved over the identically implanted Ge due to the presence of a small amount of carbon.

Manganese ions were implanted into a $250 \mathrm{~nm}$ epitaxial UHV chemical vapor deposited GeC film, grown on a Si (100) wafer, with an energy of $20 \mathrm{keV}$ and a dose of $1.1 \times 10^{16} \mathrm{~cm}^{-2}$ at room temperature and subsequently annealed at various temperatures and time. The structure of the GeCMn films was characterized by TEM and grazing incidence XRD (GI-XRD). The objective of this study is to report on the structural properties as determined by TEM of ion implanted GeMn grown on Si.

Bright field and high resolution TEM images of as-implanted samples, $300{ }^{\circ} \mathrm{C}$ and $500{ }^{\circ} \mathrm{C}$ annealed samples are shown in Figures 1, 2 and 3, respectively. Nano-beam diffraction patterns taken from the $\mathrm{Mn}$ implanted $\mathrm{GeC}$ regions were also presented as inserts. A clear crystalline/amorphous interface can be observed in all 3 cases and $\mathrm{GeC}$ film epitaxial grew along Si (001) wafer. The HRTEM images and nano-diffraction patterns show that the Mn-implanted $\mathrm{GeC}$ layer are all amorphous, even after $500{ }^{\circ} \mathrm{C}$ annealing. It is worth noting that the medium range order appearing in the nano diffraction patterns in the amorphous Mn-doped $\mathrm{GeC}$ increases with increasing annealing temperature. The amorphous features have also been confirmed by the GI-XRD patterns shown in Figure 4. The Ge crystalline peaks from the $500{ }^{\circ} \mathrm{C}$ annealing sample might be from the reorganization of dislocations and formation of subgrains from the sublayer GeC (shown in Figure 5) [5].

References:

[1] H. Ohno, Science 281, 951 (1998).

[2] N. Theodoropoulou et al., J. Appl. Phys. 91, 7499 (2002).

[3] M. Bolduc et al., Phys. Rev. B 71, 033302 (2005).

[4] A. Verna et al., Phys. Rev. B 74, 085204 (2006).

[5] This work was supported by the SWAN program funded by the GRC-NRI. 


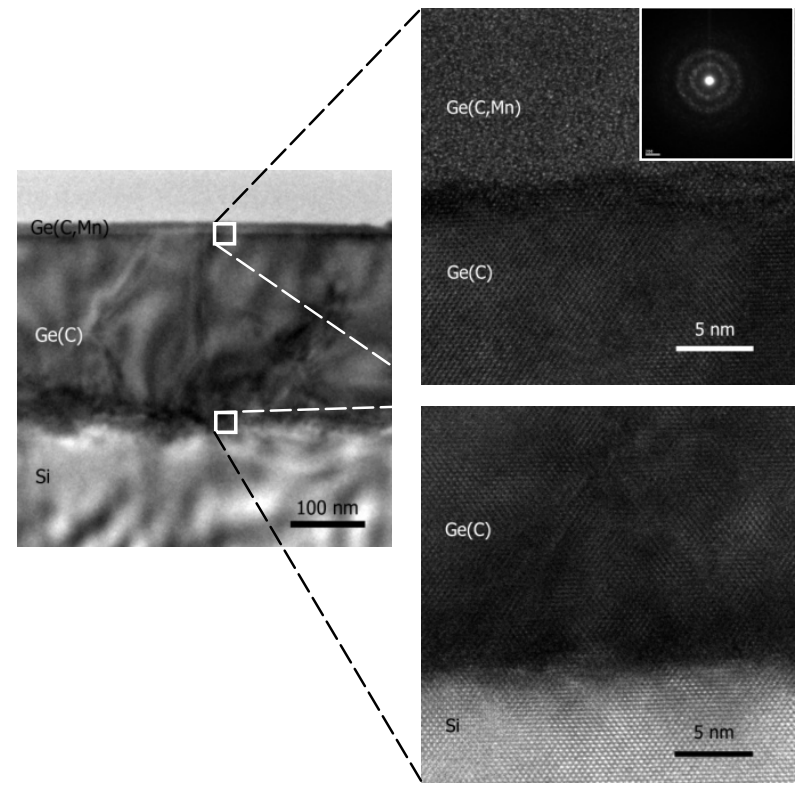

Fig. 1. TEM cross-section of the as-implanted GeCMn.

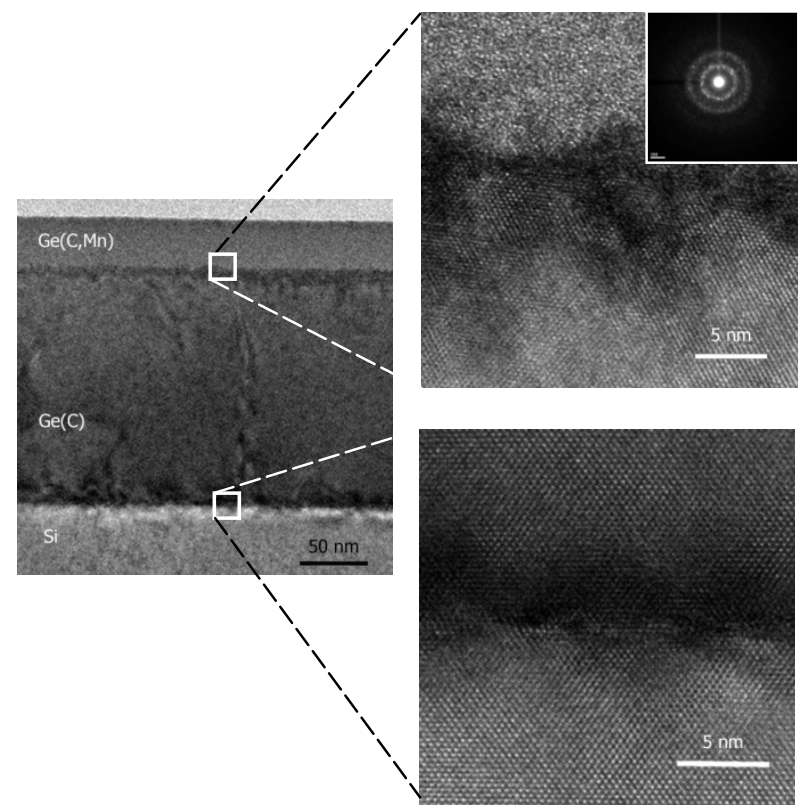

Fig. 3. TEM cross-section of the $500{ }^{\circ} \mathrm{C}$ annealed sample.
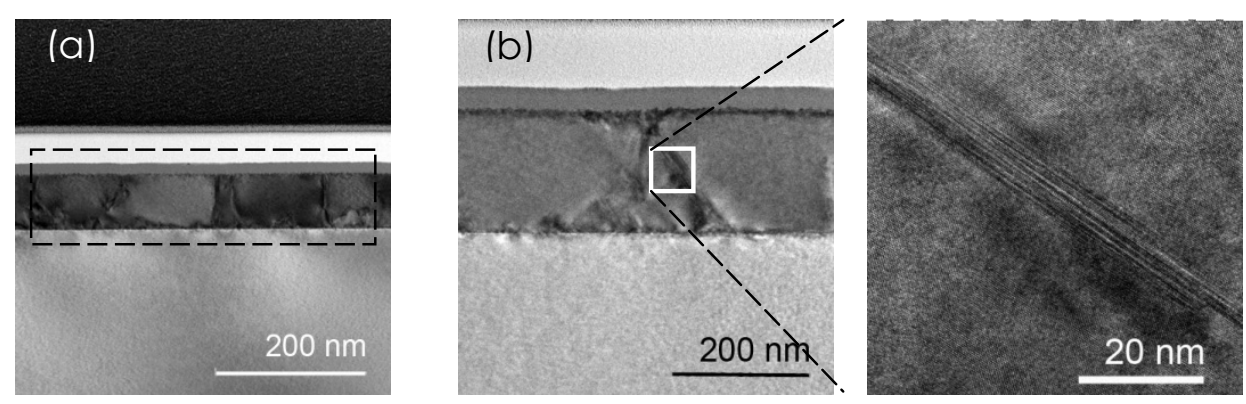

Fig. 5. (a) Dislocations and grain boundaries, and (b) and micro-twin structure in the $500^{\circ} \mathrm{C}$ annealed sample.

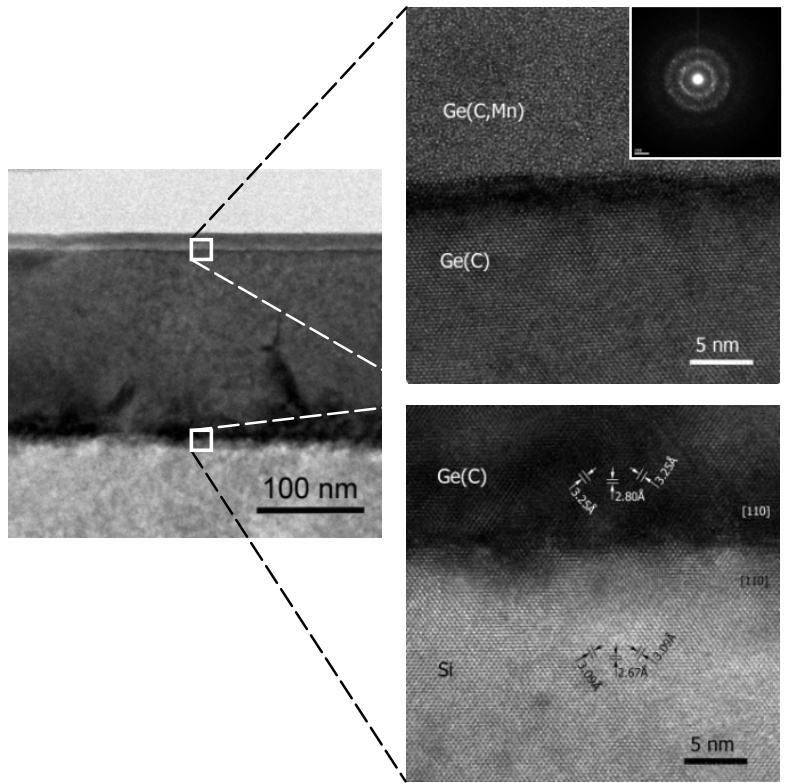

Fig. 2. TEM cross-section of the $300{ }^{\circ} \mathrm{C}$ annealed sample.

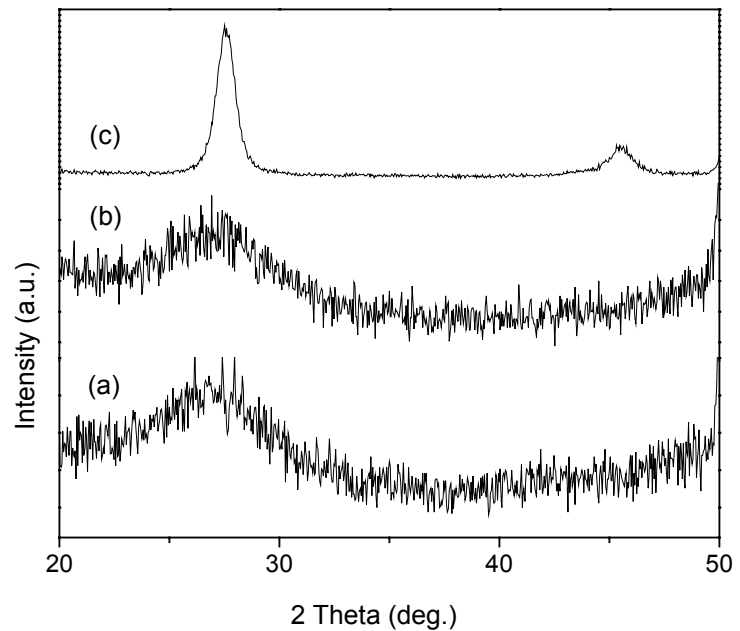

Fig. 4. Grazing incidence $\left(0.3^{\circ}\right) \mathrm{XRD}$ spectra: (a) asimplanted (b) $300^{\circ} \mathrm{C}$ and (c) $500^{\circ} \mathrm{C}$ annealed samples. 